\title{
Estudios socio-ecológicos de largo plazo en los sitios fundadores de la red LTSER-Chile: desafíos y oportunidades para el futuro
}

\author{
Long term socio-ecological research of the three LTSER-Chile founder sites: \\ challenges and opportunities for future research
}

\author{
Aurora Gaxiola a,b*, Juan Luis Celis-Diez c, Ricardo Rozzi a,d,e , Julio Gutiérrez a,f,g \\ *Autor de correspondencia: a Instituto de Ecología y Biodiversidad, Las Palmeras 3425, Ñuñoa, \\ Santiago, Chile, tel. 2354 2976, agaxiola@uc.cl \\ ${ }^{b}$ Pontificia Universidad Católica de Chile, Departamento de Ecología, Alameda 340, Santiago, Chile. \\ c Pontificia Universidad Católica de Valparaíso, Escuela de Agronomía, Calle San Francisco S/N, La Palma, Quillota, Chile. \\ Centro de Desarrollo Urbano Sustentable, CEDEUS, Santiago, Chile. \\ ${ }^{\mathrm{d}}$ University of North Texas, Sub-Antarctic Bio Cultural Conservation Program, Denton, Texas, USA. \\ ${ }^{\text {e } U n i v e r s i d a d ~ d e ~ M a g a l l a n e s, ~ P a r q u e ~ E t n o b o t a ́ n i c o ~ O m o r a, ~ P u n t a ~ A r e n a s, ~ C h i l e . ~}$ \\ ${ }^{\mathrm{f}}$ Universidad de La Serena, Benavente 980, La Serena, Chile. \\ ${ }^{\mathrm{g}}$ Centro de Estudios Avanzados en Zonas Áridas, Raúl Bitrán 1305, La Serena, Región de Coquimbo, Chile.
}

\section{SUMMARY}

The research carried out in the three foundational sites of the Chilean Long-Term Socio-Ecological Research Network (LTSER-Chile) aims to answer questions that reflect the regional context in which each site is immersed. The formation of the network in 2008 provided an opportunity to establish comparative studies and analyse the effects of the different components of global change could exert on different ecosystems in Chile. This study presents an analysis of the research programs of each of the three sites and presents the context in which these lines have been established. Each founder site has identified key aspects associated with local needs and the interests of the research groups that initiated and have directed the ecological or socio-ecological long-term studies. At the national scale these different approaches are complementary. First, we present an analysis and evaluation of the research conducted by each of these groups. Then, we develop a diagnosis of the research developed at each of the sites during the last two decades with the goal of identifying gaps and future research opportunities. Furthermore, this characterization will contribute to addressing the challenges and opportunities in basic and applied long-term socio-ecological research.

Key words: global change, climatic change, Cape Horn, Chiloé, Fray Jorge National Park.

\section{RESUMEN}

La investigación que se desarrolla en los tres sitios fundadores de la red Chilena de Sitios de Estudios Socio-Ecológicos de Largo Plazo (LTSER-Chile) intenta responder preguntas que reflejan el contexto regional de cada sitio. La conformación de la LTSER-Chile en 2008 ofrece una oportunidad para generar estudios comparativos y analizar los potenciales efectos del cambio socio-ambiental global en los diferentes ecosistemas de Chile. Este estudio presenta un análisis de las líneas de investigación desarrolladas en cada uno de los tres sitios y el contexto en el que se han establecido. Cada sitio fundador ha identificado aspectos claves de necesidades locales y del interés de los grupos de investigadores que han iniciado y dirigido los estudios ecológicos o socio-ecológicos a largo plazo. A nivel nacional estos diversos enfoques resultan complementarios. Primeramente, se presentan los análisis y evaluaciones que realizan cada uno de estos grupos de investigación, y luego, un breve diagnóstico del trabajo de las últimas dos décadas en los sitios que actualmente son parte de la LTSER-Chile, con el fin de identificar vacíos y oportunidades de investigación. Asimismo, esta caracterización contribuirá a abordar los retos y oportunidades en investigación socio-ecológica de largo plazo, básica y aplicada.

Palabras clave: cambio global, cambio climático, Cabo de Hornos, Chiloé, Fray Jorge.

\section{INTRODUCCIÓN}

Los sistemas ecológicos están sujetos a cambios continuos en el número y tipo de especies que los componen, en la complejidad de la estructura de las relaciones intra- $\mathrm{e}$ inter-específicas que los mantienen y caracterizan, como también en la disponibilidad temporal y espacial de recursos bióticos y abióticos que los mantienen. Por lo tanto, los estudios ecológicos de largo plazo permiten identificarla dinámica natural de los ecosistemas, su principales factores de cambio y capacidad de respuesta y resiliencia (Likens1989, Armesto 1990, Knapp et al. 2012). Una importancia central de la investigación ecológica de largo plazo es la ampliación de las escalas temporales y espaciales, en las cuales, estudios de corta duración (menos de tres años) adquieren una nueva perspectiva (Armesto 
1990). La falta de una perspectiva histórica puede determinar que estudios de corta duración caigan en un "presente invisible" (Magnuson 1990); es decir, cuando los cambios observados en un tiempo determinado están modulados por estados previos del sistema (Armesto 1990). Por lo tanto, los datos de largo plazo generan una escala sintonizada a las condiciones espacio-temporalmente contextualizadas de un sistema. Por ejemplo, en el centro y norte de Chile los años 1997 y 1998 han sido los más y menos lluviosos, respectivamente, en los últimos 30 años (Jiménez et al. 2011). Por lo tanto, estudios que se hayan realizado en estos años por separado o durante los dos años, representarán condiciones muy particulares y acotadas de este ecosistema. El enfoque de largo plazo contribuye a resolver esta limitación al ampliar la escala espacio-temporal en donde se puede identificar a los años 1997 y 1998 como fuera de la norma y evaluarlas implicaciones ecológicas de estas fluctuaciones (Meserve et al. 2003).

El monitoreo es un componente clave de los estudios de largo plazo, ya que proporcionan la línea base sobre la cual se puede comparar y evaluar la naturaleza del cambio en los sistemas ecológicos (Lindenmayer y Likens 2009). El monitoreo permite entender y cuantificarlas respuestas a las perturbaciones naturales (sequías, heterogeneidad ambiental, etc.) (Holmgren et al. 2006) y al impacto de las manipulaciones experimentales (adición de nutrientes) (Neff et al. 2002)sobre los sistemas biológicos. Finalmente, los estudios de largo plazo, además de generar conocimiento básico de los sistemas ecológicos, permiten el diseño, síntesis y ejecución de estudios comparativos que promueven la generación de programas de conservación y manejo a escala nacional e internacional (Armesto 1990, Franklin y Swanson 2010, Maass et al. 2010, Knapp et al. 2012).

El año 2008 se estableció la red LTSER-Chile (su acrónimo se alude al inglés Long-Term Socio-Ecological Research) con tres sitios fundadores: el Parque Nacional Bosque Fray Jorge (PNFJ, 30 $\mathrm{S}$ ), la Estación Biológica Senda Darwin (EBSD, $42^{\circ} \mathrm{S}$ ), y el Parque Etnobotánico Omora (PO, $\left.55^{\circ} \mathrm{S}\right)$. La LTSER-Chile se estableció a partir de los programas de investigación de estos tres sitios que representan el trabajo conjunto de investigadores que históricamente han tenido diferentes aproximaciones a la investigación ecológica de largo plazo. Los cuatro objetivos de este estudio son describir: 1) las líneas de investigación desarrolladas en cada uno de los tres sitios, 2) el contexto en el que estas líneas se han establecido y, 3) la investigación transversal que se realiza en estos sitios. Considerando que cada grupo de trabajo ha identificado aspectos claves de interés local, pero que son complementarios a nivel nacional, se presentan primero los estudios que realizan cada uno de los tres grupos de investigación, y luego se aborda el último objetivo: 4) discutir y proponer objetivos locales y generales de investigación futura en la Red LTSER-Chile.

\section{ESTUDIOS DE LARGO PLAZO EN LOS SITIOS FUNDADORES DE LA RED LTSER-CHILE}

Los tres sitios cubren desde los ecosistemas semiáridos en el PNFJ, localizado al sur del desierto de Atacama $\left(30^{\circ} \mathrm{S}\right)$, pasando por los bosques templados lluviosos y las turberas de la EBSD, en la Isla Grande de Chiloé $\left(42^{\circ} \mathrm{S}\right)$, hasta la eco-región subantártica de Magallanes en el Parque Etnobotánico Omora $\left(55^{\circ} \mathrm{S}\right)$, en la Reserva de la Biósfera Cabo de Hornos. El énfasis que se ha dado a la investigación de largo plazo en cada sitio refleja esta diversidad, como se constata en las tres síntesis de las investigaciones publicadas recientemente (Rozzi et al. 2006, Carmona et al. 2010, Gutiérrez et al. 2010). En su primera etapa de existencia, no obstante la institucionalidad adquirida por la red LTSER-Chile, la diversidad de enfoques ha marcado la aproximación de estudios a largo plazo en cada sitio. En una segunda etapa, sin embargo, se hace necesario identificar temas transversales para abordar preguntas comunes con líneas y protocolos de investigación compartidos para implementar de manera efectiva la escala espacial y ecológica que este conjunto representa. Por lo tanto, en este trabajo se presenta un diagnóstico de la investigación que se ha desarrollado en cada sitio, para identificar vacíos de investigación y oportunidades de relevancia nacional e internacional con el fin de promover estudios comparativos con otros sitios de largo plazo que existan en Chile y el mundo.

\section{INVESTIGACIÓN DE LARGO PLAZO EN EL PARQUE NACIONAL BOSQUE FRAY JORGE}

La investigación de largo plazo tiene diferentes etapas, las cuales pueden diseñarse y desarrollarse en paralelo o de forma alternada, yel monitoreo es el pilar fundamental de esta investigación (Likens 1989, Armesto 1990, Lindenmayer y Likens 2009). En el sitio PNFJ un grupo de investigadores comenzaron hace 25 años un experimento de gran escala espacial (Meserve et al. 1995). En sus inicios la aproximación desde el punto de vista de ecología de comunidades tenía como objetivos principales evaluar el papel de los micromamíferos herbívoros, las tramas tróficas y la importancia relativa de los controles bióticos y abióticos. Para abordar estos objetivos se establecieron 16 parcelas de 75 x 75 metros de exclusión de herbívoros y depredadores. Los resultados de este estudio han permitido detectar el rol clave de la variación interanual en las precipitaciones, lo cual contradice la hipótesis inicial que postulaba a las interacciones bióticas como elementos moduladores en la dinámica de estas comunidades. Es esencial notar que luego de 26 años de estudio, muchas de las hipótesis y percepciones iniciales acerca de la estructura y dinámica de este sistema semiárido de Chile, no solamente cambiaron, sino que requirieron modificaciones en la aproximación experimental y en el monitoreo (Gutiérrez et al. 2010). Este experimento ha cambiado a lo largo del tiempo bajo un enfoque 
de monitoreo adaptativo (Lindenmayer y Likens 2009). En la actualidad este estudio ecológico experimental es el de mayor escala espacial y temporal del Hemisferio Sur.

En los fragmentos de bosque templado que se ubican en la cima de la Cordillera de la Costa del PNFJ también se ha desarrollado monitoreo de largo plazo. El carácter relicto y el aislamiento de estos bosques templados representan un experimento natural para investigar las consecuencias a largo plazo de la fragmentación. Desde 1995, en esta sección del PNFJ se han evaluado los efectos del tamaño de los fragmentos en la estructura comunitaria, interacciones bióticas y dinámica poblacional de grupos de aves, insectos y especies vegetales (Cornelius et al. 2000, Barbosa y Marquet 2002, Vergara y Marquet 2007, Núñez-Ávila et al. 2013). A nivel ecosistémico, se han desarrollado estudios sobre el rol de la neblina como fuente clave agua y de la estructura espacial de los parches del bosque relicto (Del Val et al. 2006, Stanton et al. 2013). Estos estudios ecosistémicos han requerido del monitoreo constante de organismos como aves y mamíferos, y de las variables climáticas y abióticas, los cuales han permitido entender los potenciales efectos dela fragmentación y los cambios en la estructura y dinámica de los bosques sobre la pérdida de especies.

\section{INVESTIGACIÓN DE LARGO PLAZO EN LA ESTACIÓN BIOLÓGICA SENDA DARWIN}

Históricamente, la investigación de la EBSD se ha concentrado en interacciones mutualistas planta-animal, en la composición y estructura de la vegetación y comunidades de aves del bosque (Armesto et al. 1996, 2009). Los estudios sobre la dinámica de bosques son un componente esencial en la agenda de investigación de largo plazo de la EBSD (Armesto et al. 2009) así como del programa de monitoreo de especies sensibles a la pérdida y fragmentación del bosque, como el chucao, Scelorchilus rubecula (Wilson et al. 2014) y el monito de monte, Dromiciops gliroides (Celis-Diez et al. 2012). El monitoreo de los polinizadores y la fenología floral de las especies arbóreas, junto con la dinámica poblacional de vertebrados especialistas de bosques han sido utilizados para integrar la información a nivel ecosistémico sobre el impacto -en el corto y largo plazo-de la pérdida, fragmentación y degradación del bosque (Jaña-Prado et al. 2007, Armesto et al. 2009).

La investigación que se realiza en la EBSD está orientada principalmente al estudio de los bosques templados, detectando las tendencias en los ciclos de crecimiento y regeneración de las especies arbóreas e identificando los factores locales y globales que degradan estos ecosistemas. En este contexto la EBSD ha diseñado un programa de investigación que incluye monitoreo de largo plazo e intervenciones experimentales y monitoreo sobre de la dinámica y estructura de ecosistemas templados incluyendo bosques y turberas. El principal objetivo de este programa es el generar una línea de base sobre la dinámica y estructura de dichos ecosistemas, con el fin de evaluar la magnitud del efecto que tendrán los cambios en los factores abióticos (disponibilidad de nutrientes, temperaturas y precipitaciones de verano, etc.) y bióticos (mortalidad y reclutamiento) sobre estos ecosistemas (Armesto et al. 2009, Carmona et al. 2010). Finalmente, considerando el estrecho vínculo entre las comunidades locales y la dinámica de los bosques, en la EBSD se han seleccionado sitios en áreas dominadas por bosque secundario y primario para establecer réplicas de parcelas permanentes (Carmona et al. 2010). La implementación de parcelas permanentes en bosques con diferentes estados sucesionales producto de las intervenciones antrópicas, permitirá evaluar el efecto del uso y manejo tradicional sobre la dinámica de los bosques nativos (Carmona et al. 2010).

\section{INVESTIGACIÓN DE LARGO PLAZO EN EL PARQUE ETNOBOTÁNICO OMORA}

El Parque Etnobotánico Omora es el más reciente de los tres sitios fundadores. Basado en su desarrollado enfoque socio-ecológico y en la experiencia de PNFJ y EBSD, el Parque Etnobotánico Omora planteó la creación formal de la red LTSER-Chile con el doble fin de cubrir los extremos latitudinales de bosques templados en Chile $\left(30^{\circ}\right.$ a $55^{\circ}$ S) y de integrar transdisciplinariamente la investigación ecológica de largo-plazo con la toma de decisiones socioambientales por parte del estado de Chile. La integración socio-ecológica se ha forjado a través de la combinación de las ciencias ecológicas y la ética ambiental, lo cual a su vez ha ampliado el espectro disciplinario de los estudios socio-ecológicos de largo plazo a nivel mundial (Rozzi et al. 2012), ya que la integración socio-ecológica hasta ahora ha sido fundamentalmente socio-económica.

En contraste con PNFJ (terrenos públicos) y EBSD (terrenos privados), el Parque Etnobotánico Omora es una iniciativa público-privada que se desarrolla en una concesión otorgada por el Ministerio de Bienes Nacionales a la Fundación Omora y la Universidad de Magallanes. Desde el año 1999 este parque ha definido tres ámbitos de trabajo que han sido adaptados desde el enfoque de la "Iniciativa para una biosfera sustentable" (Lubchenco et al. 1991) que consiste en: a) investigación ecológica transdisciplinaria de largo plazo, b) educación formal y no formal, y c) conservación biocultural, incluyendo el turismo de intereses especiales como una forma de contribuir a la sustentabilidad ambiental, económica y social. Para desarrollar estos ámbitos de trabajo el Parque Etnobotánico Omora estableció tres áreas de investigación transdisciplinaria dividida en sublíneas (cuadro 1): (I) Biodiversidad Subantártica poco percibida (Rozzi et al. 2008a, Contador et al.2012, Arróniz-Crespo et al. 2014), (II) Investigación y monitoreo de largo plazo (Ibarra et al. 2009, Jiménez et al. 2014, Rozzi y Jiménez 2014) y (III) Integración de ética ambiental, educación y conservación biocultural (Rozzi et al. 2008b, 2013, Schüttler et al. 2011). 


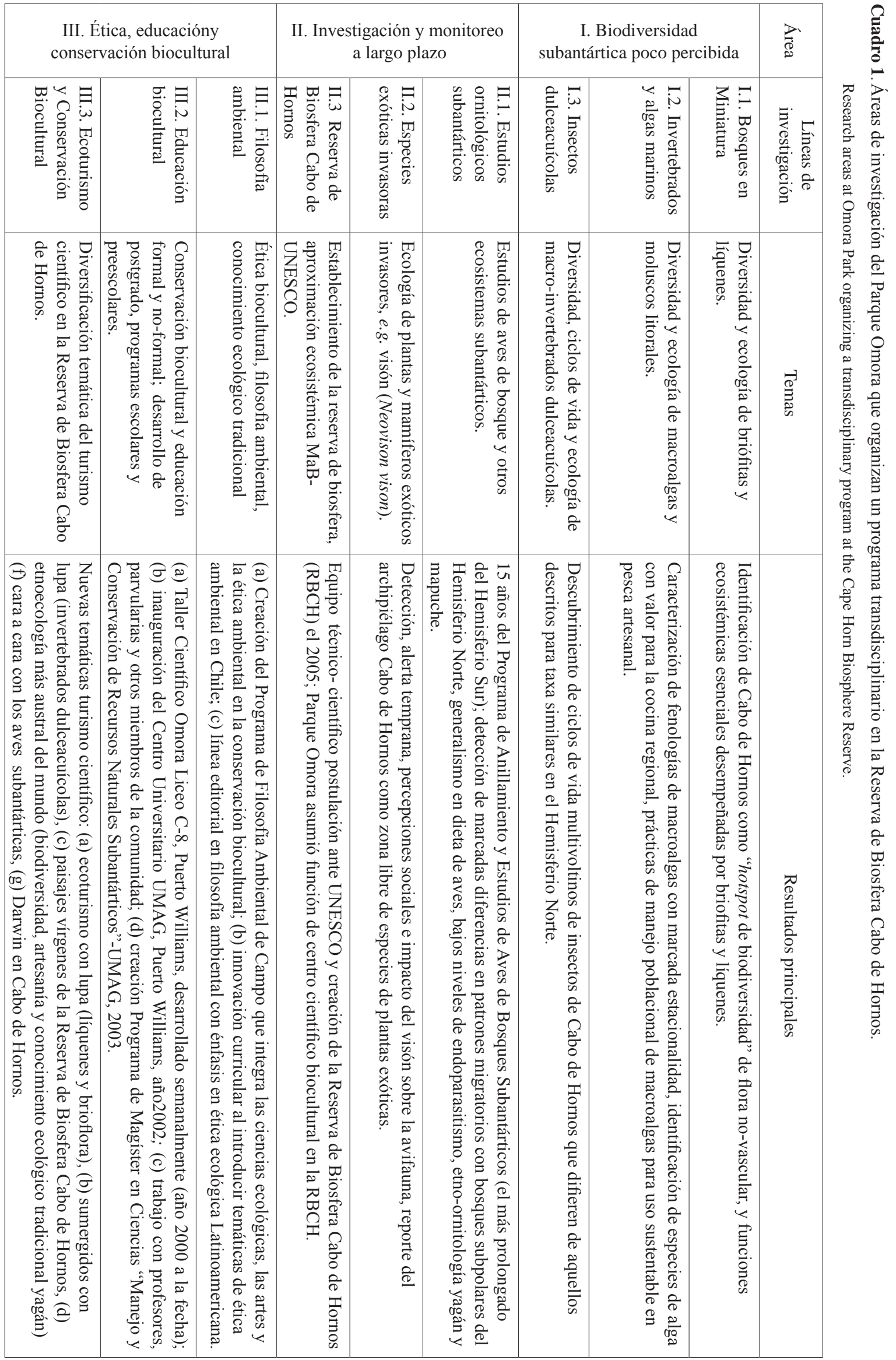




\section{COMPARACIÓN ENTRE SITIOS}

Las líneas de investigación que se han establecido de forma independiente en cada uno de los sitios fundadores de la LTSER-Chile (figura 1), representan un complemento de aproximaciones que van desde estudios de la autoecología de especies, estudios experimentales de comunidades, monitoreo de poblaciones y ecosistemas, hasta estudios de la dimensión ética, conocimiento ecológico tradicional amerindio, y sobre política y toma de decisiones.

Un primer paso para abordar preguntas y protocolos comunes ha sido establecer una red de estaciones climáticas que registran variables ambientales. La red de estaciones registra las tendencias climáticas locales dentro del contexto nacional y mundial. Los datos de las estaciones se utilizan como co-variables para entender los cambios en los patrones demográficos y ecosistémicos que se monitorean en los diferentes sitios, y generan una línea base contra la cual comparar y dar contexto a los resultados de estudios de menor duración. El objetivo general de esta red climática es generar datos que permitan identificar la susceptibilidad de los ecosistemas a los efectos del cambio climático. Finalmente, los registros climáticos de la LTSER-Chile proveen datos claves para poner a prueba diferentes hipótesis y modelos de cambio climático regional y global (Jiménez et al. 2011).

Un segundo paso ha sido la manipulación experimental con el fin de identificar en el corto plazo los efectos que los escenarios de cambio global pueden tener en la estructura de los ecosistemas. Por ejemplo, los modelos de cambio climático para el sur de Chile predicen disminuciones de aproximadamente un $30 \%$ de las lluvias estivales (DGF y CONAMA 2006). En EBSD, considerando este escenario futuro, se estableció un experimento de exclusión de lluvias de verano (Carmona et al. 2010). El experimento comenzó con el diseño y establecimiento de un sistema de registro de variables ambientales y de monitoreo de productividad primaria (caída de hojarasca, crecimiento de árboles, cambios en la estructura del dosel, etc.), para generar la línea de base que permita una mejor interpretación

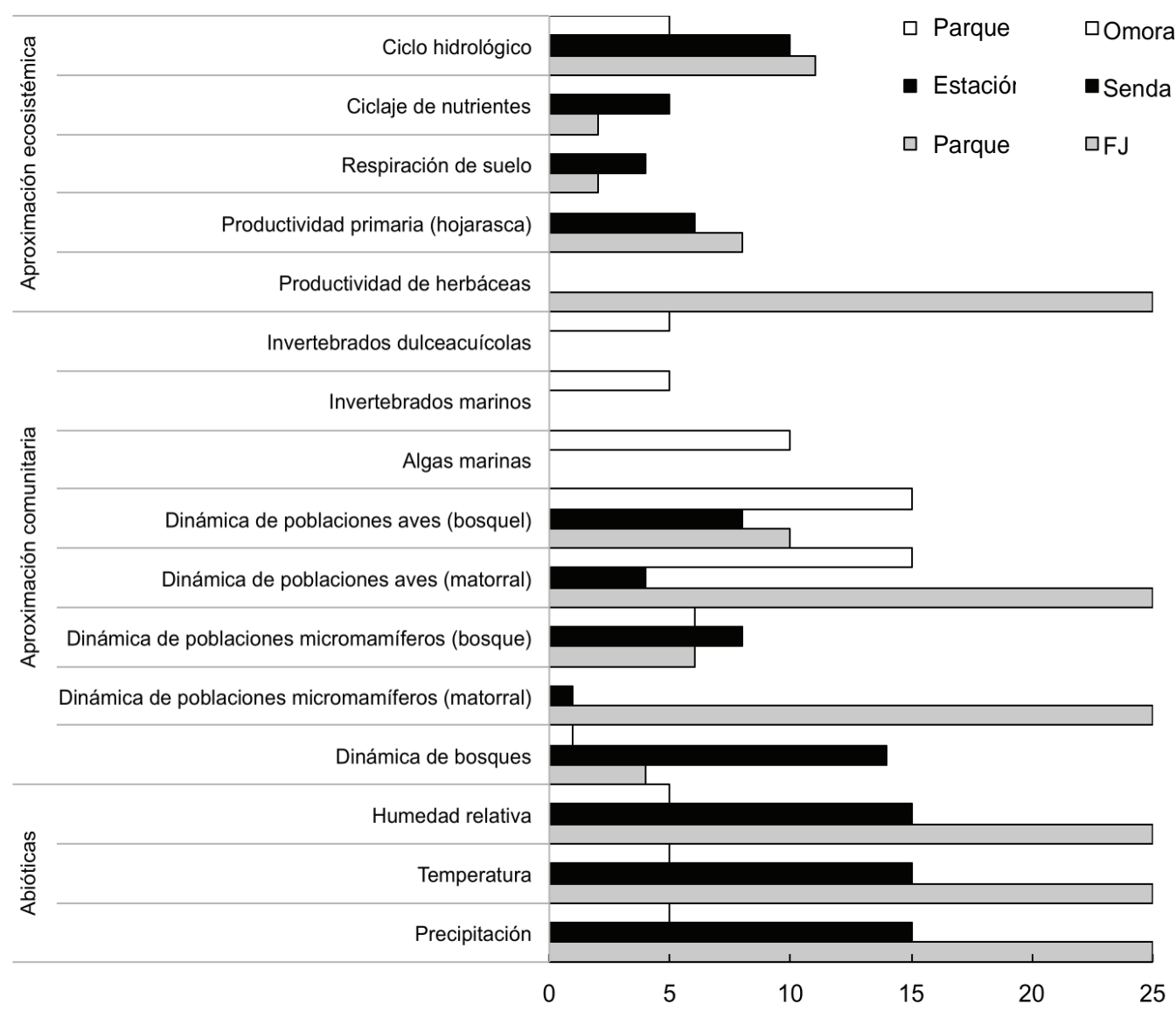

Figura 1. Estudios de largo plazo en los tres sitios fundadores de la LTSER-Chile. Las barras muestran la duración (en años) del monitoreo a las diferentes variables. Los estudios están divididos en función de la naturaleza de las variables (abióticas) y de la escala ecológica en la que se establecieron los registros y monitoreo (aproximaciones). Los colores representan a los diferentes sitios.

Long-term studies in the three LTSER-Chile founder sites. Bars show the length of duration (in years) of the records for different variables. Studies are divided in relation to the variables (abiotic) or ecological level from which the long-term monitoring began (approach). Colours represent one of the three founder sites. 
de los datos que se generen en las parcelas experimentales. El monitoreo de largo plazo junto con la manipulación experimental son herramientas de investigación que se han desarrollado en la EBSD en este segunda etapa de consolidación de la red LTSER-Chile.

En el PNFJ el monitoreo establecido en el experimento de exclusión de herbívoros y carnívoros (ver sección Parque Nacional Bosque Fray Jorge) se ha complementado con estudios experimentales de corto plazo (Gutiérrez et al. 2010). Por otro lado, dada la importancia de la neblina en el mantenimiento del bosque relicto de PNFJ (Del Val et al. 2006), se ha refinado la escala temporal y espacial de las mediciones de neblina. En el Parque Etnobotánico Omora se ha consolidado un programa de 15 años monitoreo de aves del bosque templado, comenzando con elmétodo de anillamiento, captura y recaptura, complementándose recientemente con geolocalizadores para estudiar las rutas de las especies migratorias. Estos estudios tienen objetivos específicos dentro de los grandes objetivos generales de investigación de largo plazo de la LTSER-Chile, que es evaluar y detectar los potenciales efectos del cambio global sobre los ecosistemas.

Un tercer paso ha sido el establecimiento de parcelas permanentes para monitorear la dinámica de los bosques templados a lo largo de su gradiente latitudinal de distribución geográfica, bajo condiciones biofísicas y antrópicas contrastantes, junto con estudiar los procesos biogeoquímicos e hidrológicos que los caracterizan. El contexto local de cada una de estas parcelas permitirá analizar las consecuencias y los impactos que la pérdida de la biodiversidad o diferentes tipos de uso y manejo, generan sobre las funciones ecosistémicas.

Durante el primer quinquenio de existencia de la LTSER-Chile, esta complementariedad favoreció la creatividad para establecer estudios ecosistémicos pioneros en la evaluación de procesos bióticos y abióticos (Gutiérrez et al. 2010, Carmona et al. 2010), y en la integración transdisciplinaria de la ética ambiental (Rozzi et al. 2013).

\section{OPORTUNIDADES Y DESAFÍOS FUTUROS EN LA RED LTSER-Chile}

El desafío para el siguiente quinquenio es lograr una mayor articulación en la investigación entre sitios con el fin de abordar preguntas comparativas que aprovechen el rango de variación en precipitaciones, temperatura y fotoperíodo, así como de la influencia antropogénica. Asimismo, la LTSER-Chile tiene como desafío la integración de las ciencias ecológicas y la filosofía ambiental en la investigación socioecológica de largo plazo. El marco conceptual de la ética biocultural que define tres dominios de investigación transdiciplinaria: biofísico, simbólico-lingüístico e institucional socio-político (Rozzi et al. 2013) es una oportunidad que tiene la LTSER-Chile. Un resultado de esta integración es la conceptualización y documentación del fenómeno de homogeneización biocultural, en donde actividades pro- ductivas y de educación son monopolizadas por unas pocas especies, lenguajes o valores culturales empíricos (Rozzi et al. 2008b). Para revertir la homogeneización biocultural, el Parque Etnobotánico Omora ha establecido programas de educación y conservación biocultural en la Reserva de Biosfera Cabo de Hornos que podrían extenderse hacia los demás sitios. La aproximación metodológica de la "filosofía ambiental de campo" (Rozzi et al. 2012) ha sido adoptada como una metodología estructurada para el diseño de mallas curriculares y tesis, generando actividades innovadoras de educación y conservación biocultural, incluyendo el ecoturismo. Se espera que en este segundo quinquenio esta metodología pueda desarrollarse en los sitios fundadores y en los nuevos sitios asociados a la red LTSER-Chile.

\section{CONCLUSIONES}

Las características ecológicas y culturales de los sitios que actualmente conforman la red LTSER-Chile han permitido el diseño de preguntas y estudios únicos, que dentro del contexto de los estudios ecológicos de largo plazo, han generado información de relevancia nacional e internacional para cada bioma, ecosistema o región específica. Durante el segundo quinquenio de la red LTSER-Chile se trabajará en resolver vacíos en investigación y en promover oportunidades para el desarrollo de nuevas preguntas. Finalmente, la LTSER-Chile está promoviendo la incorporación de nuevos sitios a la red, que han desarrollado estudios de largo plazo en Chile lo que permitirá aumentar la representatividad de los ecosistemas, desarrollar nuevas preguntas de investigación, aproximaciones metodológicas y potenciar la investigación transdisciplinaria. Se espera que una mejor integración de la investigación de largo plazo en Chile ofrezca nuevas oportunidades para implementar cursos de campo para estudiantes, investigadores post-doctorales y jóvenes científicos. Para este fin, el análisis de las líneas de investigación y el trabajo desarrollado en los sitios de la LTSER-Chile constituye un ejercicio de análisis que permite identificar vacíos y oportunidades de investigación futura.

\section{REFERENCIAS}

Armesto JJ. 1990. Estudio a largo plazo: Una prioridad para la investigación ecológica de hoy. Revista Chilena de Historia Natural 63: 7-9.

Armesto JJ, R Rozzi. 1989. Seed dispersal syndromes in the rain forest of Chiloé: evidence for the importance of biotic dispersal in a temperate rain forest. Journal of Biogeography 219-226.

Armesto JJ, C Smith-Ramirez, C Sabag. 1996. The importance of plant-bird mutualisms in the temperate rainforest of southern South America. In Lawford RG, PB Alaback, E Fuentes eds. High-Latitude Rainforests and Associated Ecosystems of the West Coast of the Americas: Climate, Hydrology, Ecology, and Conservation. New York, USA. Springer. p. 248-265. 
Armesto JJ, M Carmona, C Smith-Ramírez, JL Celis-Diez, I Díaz, A Gaxiola, AC Gutiérrez, M Núñez-Ávila, C Pérez, R Rozzi. 2009. Old-growth temperate rain forests of South America: Conservation, plant-animal interactions, and baseline biogeochemical processes. Old-growth forests: Function, fate and value. In Wirth C, G Gleixner, M Heimanneds. Ecological Studies, Vol. 207. New York, USA. Springer. p. 367-390.

Arróniz-Crespo M, S Pérez-Ortega, A de los Ríos, TGA Green, R Ochoa-Hueso, MA Casermeiro, MT de la Cruz, A Pintado, D Palacios, R Rozzi, LG Sancho, N Tysklind. 2014. The nitrogen fixation role of bryophyte-cyanobacteria associations during primary succession in recently deglaciated areas of Tierra del Fuego (Chile). PLoS ONE 9(5): e96081. doi:10.1371/journal.pone.0096081

Barbosa O, PA Marquet. 2002. Effects of forest fragmentation on the beetle assemblage at the relict forest of Fray Jorge, Chile. Oecologia 132(2): 296-306.

Carmona MR, JC Aravena, MA. Bustamante-Sánchez, JL CelisDiez, A Charrier. 2010. Estación Biológica Senda Darwin: Investigación ecológica de largo plazo en la interfase ciencia-sociedad. Revista Chilena de Historia Natural 83(1): 113-142.

Celis-Diez JL, J Hetz, PA Marín-Vial, G Fuster, P Necochea, RA Vásquez, FM Jaksic, JJ Armesto. 2012. Population abundance, natural history, and habitat use by the arboreal marsupial Dromiciopsgliroides in rural Chiloé Island, Chile. Journal of Mammalogy 93 (1): 134-148.

Cornelius C, H Cofré, PA Marquet. 2000. Effects of habitat fragmentation on bird species in a relict temperate forest in semiarid Chile. Conservation Biology 14(2): 534-543.

Contador T, JH Kennedy, R Rozzi. 2012. The conservation status of southern South American aquatic insects in the literature. Biodiversity and Conservation 21(8): 2095-2107.

Del-Val E, JJ Armesto, O Barbosa, DA Christie, AG Gutiérrez, CG Jones, KC Weathers. 2006. Rain forest islands in the Chilean semiarid region: fog-dependency, ecosystem persistence and tree regeneration. Ecosystems 9(4): 598-608.

Franklin JF, ME Swanson. 2010. Estudios ecológicos de largo plazo en bosques de Estados Unidos: Lecciones claves para su aplicación en Chile y otras regiones. Revista Chilena de Historia Natural 83(1): 185-194.

Gutiérrez JR, PL Meserve, DA Kelt, A Engilis, M Andrea. 2010. Long-term research in Bosque Fray Jorge National Park: Twenty years studying the role of biotic and abiotic factors in a Chilean semiarid scrubland. Revista Chilena de Historia Natural 83: 69-98.

Holmgren M, P Stapp, CR Dickman, C Gracia, S Graham, JR Gutiérrez, C Hice, F Jaksic, DA Kelt, M Letnic, M Lima, BC Lopez, PL Meserve, WB Milstead, GA Polis, MA Previtali, M Richter, S Sabate, FASqueo. 2006. Extreme climatic events shape arid and semiarid ecosystems. Frontiers in Ecology and the Environment.4: 87-95.

Ibarra JT, L Fasola, DW Macdonald, R Rozzi, C Bonacic. 2009. Invasive American mink in wetlands of the Cape Horn Biosphere Reserve, Southern Chile: what are they eating? Oryx 43(1): 87-90.

Jaña-Prado R, JL Celis-Diez, AC Gutiérrez, C Cornelius, JJ Armesto JJ. 2007. Diversidad en bosques fragmentados de Chiloé: ¿Son todos los fragmentos iguales? In Grez AA, J Simonetti, RO Bustamante eds. Biodiversidad en ambientes fragmentados de Chile: Patrones y procesos en diferentes escalas. Santiago, Chile. Editorial Universitaria. p. 1591-89.

Jiménez MA, FM Jaksic, JJ Armesto, A Gaxiola, PL Meserve, DA Kelt, JR Gutiérrez. 2011. Extreme climatic events change the dynamics and invasibility of semi-arid annual plant communities. Ecology Letters (12): 1227-1235.

Jiménez JE, RD Crego, GE Soto, I Román, R Rozzi, PM Vergara. 2014. Potential impact of the alien American mink (Neovisonvison) on Magellanic woodpeckers (Campephilus magallanicus) in Navarino Island, southern Chile. Biological Invasions 16: 961-966.

Knapp AK, MD Smith, SE Hobbie, SL Collins, TJ Fahey, GJA Hansen, DA Landis. 2012. Past, present, and future roles of long-term experiments in the LTER network. BioScience 62(4): 377-389.

Likens GE. 1989. Long-Term Studies in Ecology: Approaches and Alternatives. New York, USA. Springer. 214 p.

Lindenmayer DB, GE Likens. 2009. Adaptive monitoring: a new paradigm for long-term research and monitoring. Trends in Ecology and Evolution 24(9): 482-486.

Lubchenco J, AM Olson, LB Brubaker, SR Carpenter, MM Holland, SP Hubbell. 1991. The sustainable biosphere initiative: an ecological research agenda. Ecology 72(2): 317-412.

Magnuson JJ. 1990. Long-term ecological research and the invisible present. BioScience 495-501.

Maass M, R Díaz-Delgado, P Balvanera, A Castillo, A MartínezYrízar. 2010. Redes de Investigación Ecológica y SocioEcológica a Largo Plazo (LTER y LTSER) en Iberoamérica: Los casos de México y España. Revista Chilena de Historia Natural 83(1): 171-184.

Meserve PL, JA Yunger, JR Gutiérrez, LC Contreras, WB Milstead, BK Lang, KL Cramer. 1995. Heterogeneous responses of small mammals to an El Niño Southern Oscillation Event in north central semiarid Chile and the importance of ecological scale. Journal of Mammalogy 76 (2): 580-595.

Meserve PL, DA Kelt, WB Milstead, JR Gutiérrez. 2003. Thirteen years of shifting top-down and bottom-up control. BioScience 53(7): 633-646.

Núñez-Ávila MC, M Uriarte, PA Marquet, JJ Armesto. 2013. Decomposing recruitment limitation for an avian-dispersed rain forest tree in an anciently fragmented landscape. Journal of Ecology 101(6): 1439-1448.

Rozzi R, F Massardo, C Anderson, K Heidinger, J Silander. 2006. Ten Principles for Biocultural Conservation at the Southern Tip of the Americas: The Approach of the Omora Ethnobotanical Park. Ecology and Society 11(1): 43.

Rozzi R, J Armesto, B Goffinet, W Buck, F Massardo, J Silander, M Kalin-Arroyo, S Russell, CB Anderson, L Cavieres, JB Callicott. 2008a. Changing lenses to assess biodiversity: patterns of species richness in sub-Antarctic plants and implications for global conservation.Frontiers in Ecology and the Environment 6 (3): 131-137.

Rozzi R, X Arango, F Massardo, C Anderson, K Heidinger, K Moses. 2008b. Field Environmental Philosophy and Biocultural Conservation: The Omora Ethnobotanical Park Educational Program. Environmental Ethics 30: 325-336.

Rozzi R, JJ Armesto, JR Gutiérrez, F Massardo, GE Likens, CB Anderson, A Poole, KP Moses, E Hargrove, AO Mansilla, JH Kennedy, M Willson, K Jax, CG Jones, JB Callicott, MTK Arroyo. 2012. Integrating Ecology and Environmental Ethics: Earth Stewardship in the Southern end of the 
Americas. BioScience 62(3): 226-236.

Rozzi R, STA Pickett, C Palmer, JJ Armesto y JB Callicott. 2013. Linking Ecology and Ethics for a Changing World. Values, Philosophy, and Action. Dordrecht, The Netherlands. Springer. 377 p.

Rozzi R, JE Jiménez. 2014. Magellanic Sub-Antarctic Ornithology: First Decade of Long-Term Bird Studies at the Omora Ethnobotanical Park, Cape Horn Biosphere Reserve, Chile. Denton TX, USA - Punta Arenas, Chile. University of North Texas Press - Ediciones Universidad de Magallanes. 364 p.

Schüttler E, R Rozzi, K Jax. 2011. Towards a societal discourse on invasive species management: a case study of public perceptions of mink and beavers in Cape Horn. Journal for Nature Conservation 19: 175-184.

Stanton DE, B Salgado-Negret, JJ Armesto, LO Hedin. 2013. Forest patch symmetry depends on direction of limiting resource delivery. Ecosphere 4 (5): art65.

Vergara P, PA Marquet. 2007. On the seasonal effect of landscape structure on a bird species: the thorn-tailed rayadito in a relict forest in northern Chile. Landscape Ecology 22(7): 059-1071.

Willson MF. 2004. Loss of habitat connectivity hinders pair formation and juvenile dispersal of chucaotapaculos in Chilean rainforest. The Condor 106: 166-171.

Recibido: 10.07 .14

Aceptado: 14.10.14 